\title{
Reconstruction Research of Heavy Truck Left Panel Lining Surface Based on Reverse Engineering
}

\author{
Zhou Ping ${ }^{1, a}$, Shi Yongfang ${ }^{2, b}$ \\ ${ }^{1}$ Institute of Xinjiang Construction of Vocational and Technical, Urumqi, China \\ ${ }^{2}$ The School of Medical Engineering, Xinjiang Medical University, Urumqi, China \\ a1337198971@qq.com, bonlyxjsyf@ 126.com
}

Keywords: Reverse engineering, ATOS optical scanners, Character lines, surface reconstruction, error analysis

\begin{abstract}
The author scanned the surface of left panel lining of JieFang heavy truck(J6) using ATOS optical scanner and obtained three-dimensional(3-d) point cloud data. Then realized 3-d model reconstruction of the left liner using surface modeling function of UG NX software after processing the point cloud data measured with Imageware software. Finally, the precision of 3-d model were tested. Through example, "character lines"-centered surface reconstruction ideas were studied and explored for large covered liner parts.
\end{abstract}

\section{Introduction}

Along with the wide application of computer technology in manufacturing field, especially the rapid development of digital measurement technology, product modelling technology based on the measured data become the main focus of the reverse engineering technique.

The key technology of reverse engineering is to reconstruct model for complex curved surface, i.e., measuring a material object or physical model with certain measurements to reconstruct the CAD model of the physical object by 3D geometric modelling method based on the measured data.

First, the geometrical characteristics of the prototype parts should be extracted from measured data. Namely, measured data should be segmented according to their geometrical properties, to get design and machining features of prototypal parts by the method of geometric feature matching and recognition. Then, input the split 3D data into CAD system for surface fitting, to obtain the surface CAD model of prototypal workpiece through joining the pieces together.

The reconstructed CAD model needs inspection and correction. In other words, it is needed to work out the sample according to the CAD model, and to test whether the CAD model reconstructed meet the precision or other performance target in experiments.

\section{Discussed problems}

Mainly by application of reverse engineering technology and principle, in this paper, method and strategy of data point collection, as well as measurement planning, were discussed through the study of free curve and the shape of free surface characteristics and mathematical properties, and the related methods and techniques was put forward concerning measurement point collection of free curve and curved surface. On this basis, using the principle of measuring planning and data points acquisition strategy combined with the actual, as well as collecting and measuring the actual surface data of a sample which has a free surface on a 3-coordinate measuring machine, the digital information of the sample, or "point cloud", was got. Then input it into a computer system and use software "Imageware" to pre-treat the data points "point cloud". And finally get the physical sample's free surface model by means of UG software.

\section{The data acquisition}

Scanning process and getting point cloud data. This example uses ATOS optical scanner (ATOS I $600 \mathrm{EU}$ ) of German GOM company for data collection, measurement accuracy is 
$0.1 \mathrm{~mm} / 0.5 \mathrm{~m}$. Scanning mode: grating principle and principle of GPS positioning. In order to guarantee the measurement point accuracy of the measured samples, samples should be re-wipe clean thoroughly with cleaning solvent such as water before being placed on the workbench and the actual measurement, to ensure that the dust and dirt on the surface of them without affecting the measurement accuracy.

Firstly, since the color of the lining board is gray and shining, coating imaging agent should be sprayed on the plate surface according to the measurement requirements, so you can avoid scanning distortion due to reflection on the surface of the parts, and as well increase the imaging of the surface. Besides, some reference point should be adhered to scan surface of the parts for scanner to identify and for different scans to be seamless spliced. Generally, an object can not be scanned completely for all the surface data only by one scan but by many times of scan. By positioning the common three scanning points on different scans, they can get seamless spliced. As shown in figure

Secondly, the calibration of ATOS optical scan system is divided into hardware calibration and software calibration. Hardware calibration includes mainly adjusting the aperture of the lens, focus and the focal length, for each CCD camera to be well for working, in order to get the best imaging effect. Software calibration is to choose the appropriate debugging board, and measure in thirteen different azimuths, to build a benchmark for scanning system space orientation. Normally standards need calibrating. The rapid calibration can be used when the error of reference point is repeatedly greater than $0.1 \mathrm{~mm}$ in the process of scanning. As shown in figure 1.

General standard calibration when multiple occurrences of reference point in the process of scanning fast calibration can be used when the error is greater than $0.1 \mathrm{~mm}$. As shown in figure 2 .

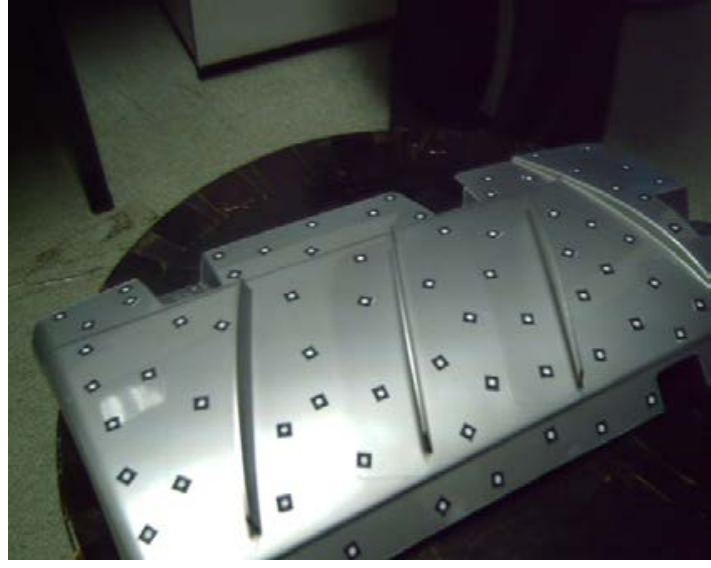

Fig. 1 left plate after reference point adhered

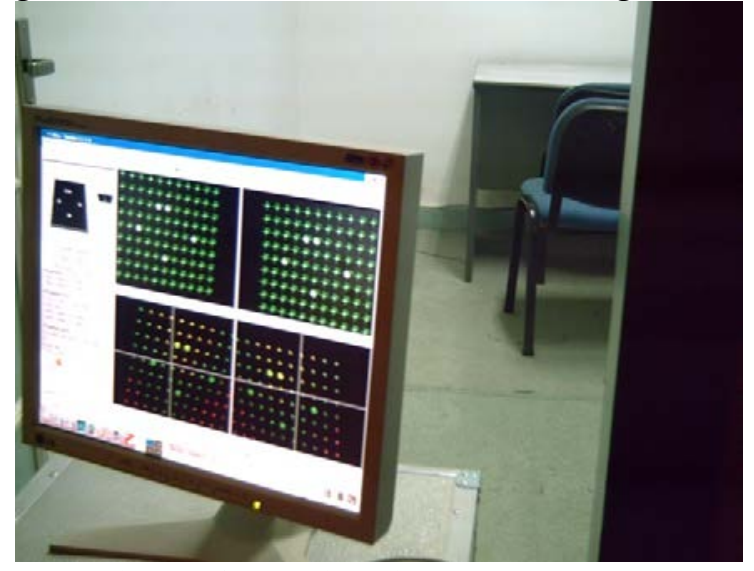

Fig. 2 fast calibration chart

ATOS data preprocessing. Alignment, triangulation, filling holes, getting rid of the noise, and smoothing and such processes of point cloud data are needed after the scan is complete by ATOS scanning software.

i) the noise data removal

ii) coordinate alignment processing

iii) the triangle calculation

iv) hole repairing of scattered data

Figure 3 showed the holes formed when reference point being adhered .

v) data smoothing

vi) output the measurement data

Finally, according to the shape of the left liner, scan it in multiple angle and different azimuths. On the basis of reference point, scanning software automatically flatten each piece of picture and eventually complete scans of the entire shape. In Figure 4 it is showed that after the scan is complete and through alignment, triangulation, diluting, smoothing and such pre-treatments, the obtained left liner shape point cloud files is output as "*.STL" files for the imageware software to treat point cloud later. 


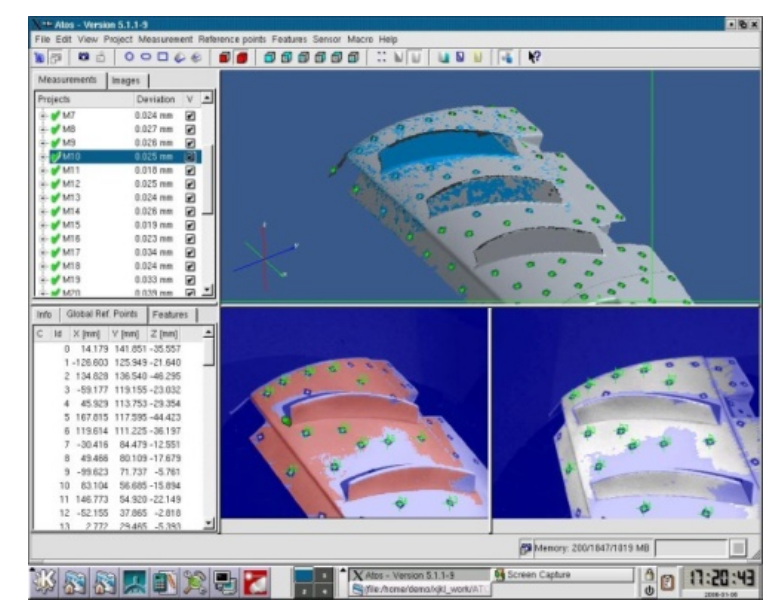

Fig.3 holes on left lining panel formed with reference point adhering

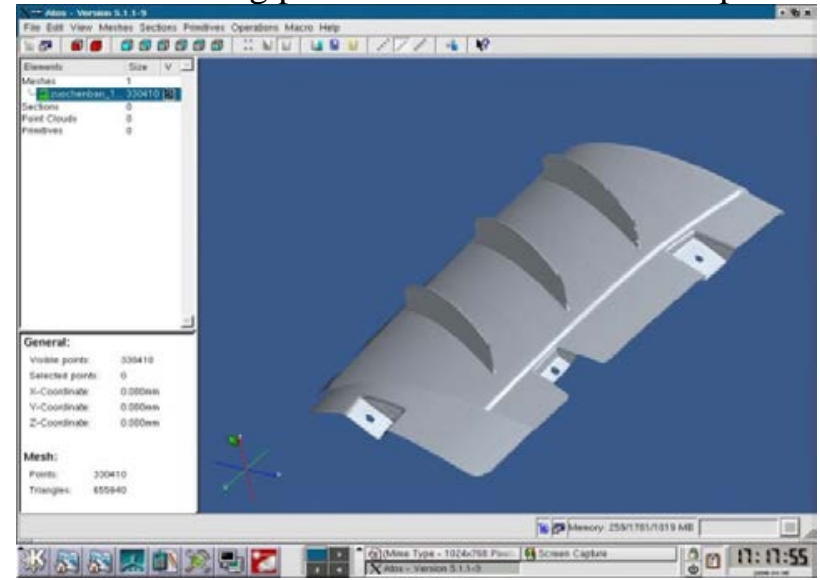

Fig. 4 left lining panel point cloud after pretreatments

The model reconstruction. The large-scale "point cloud" data modeling methods popular nowadays are far from applicable directly in existing CAD systems. Reverse engineering software's main role is to receive product data from a measuring device, and to obtain curves or surfaces models of high quality by a series of editing operations, and to transmit the curve or curved surface data to an existing CAD/CAM system by standard data format to complete the final product modelling in the system. In this case, UG uses Imageware as the software to complete reverse engineering modelling in UG series products.

Alignment of point cloud data. Configure the Imageware software for the point cloud file obtained by scanning, and according to the characteristics of point clouds, make some auxiliary benchmarks for azimuth alignment of the lining board point cloud, to prepare for extraction section line.

To do this, you should create a reference datum plane in first, and then create the corresponding alignment plane. After these, use the Imageware's Modify/Align/Stepwise function to align point cloud data with the reference datum plane.

The extraction of characteristic line. According to left plate shape characteristic, you should line out areas of quadric surface on it. And the quadric surfaces should be fitted to construct plane, cylinder or sphere, or directly make out characteristic line. Plane can be determined by three points or two intersecting straight lines. For free-form surfaces, it is necessary to construct surface characteristic line. Firstly make out the necessary section line of lining plate point cloud, then eliminate point splinters miscellaneous points on section point cloud for necessary smoothing, finally synthesize section point clouds into curve, in order to construct free-form surface.

The extraction of section feature point cloud is a frequently used method. The key link is how to find out the cross section which perpendicular to the long axis of the lining panel to obtain the best cross section shape. For the point cloud data of regular and orderly scan line, one of the line data could be directly extracted as section points. While for scattered point cloud data, if a part of it 
basically distributes regularly, for example along a straight line or circular arc, then the wanted section point could be obtained by parallel section-cutting. If the point cloud is mainly along a space curve, only a few scattered around, the space curve could be made out first, before being cut by the section perpendicular to it. This method is adopted in extraction of left plate three chamfered edge section point cloud. As shown in figure 5.

For the extracted characteristic line point cloud, it is needed firstly to use "Construct/Cross Section/Cloud Parallel" function to make out the necessary section line of the liner point clouds, and for the section line point clouds to fit a curve. Secondly, use the "Construct/Feature Line/Sharp Edges” to pick up the boundary of lining plate point cloud, and then combined the point clouds to synthetize a curve, as the basis to structure free surface. During the building process to ensure the accuracy of cross section lines and boundary, must check the deviation between point clouds and curve at any time, to guarantee the deviation of characteristic line within $0.3 \mathrm{~mm}$.

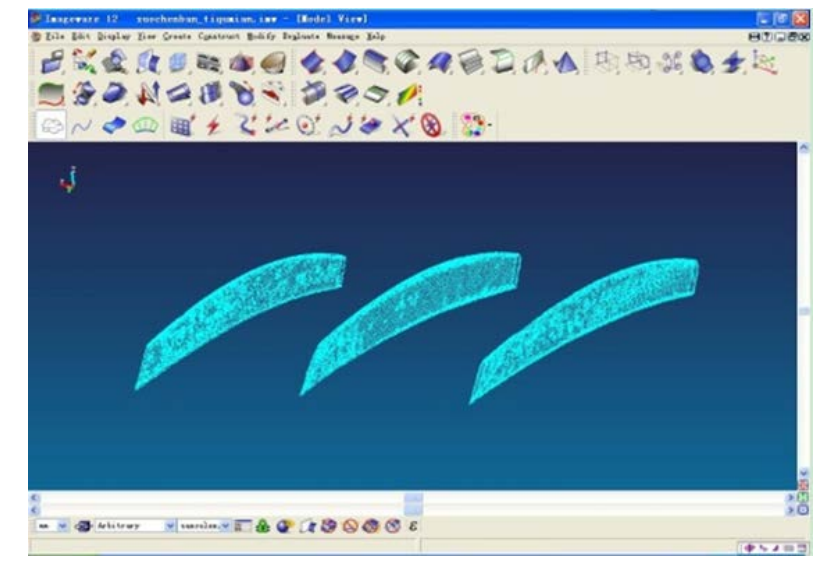

Fig. 5 three chamfered edge section point cloud

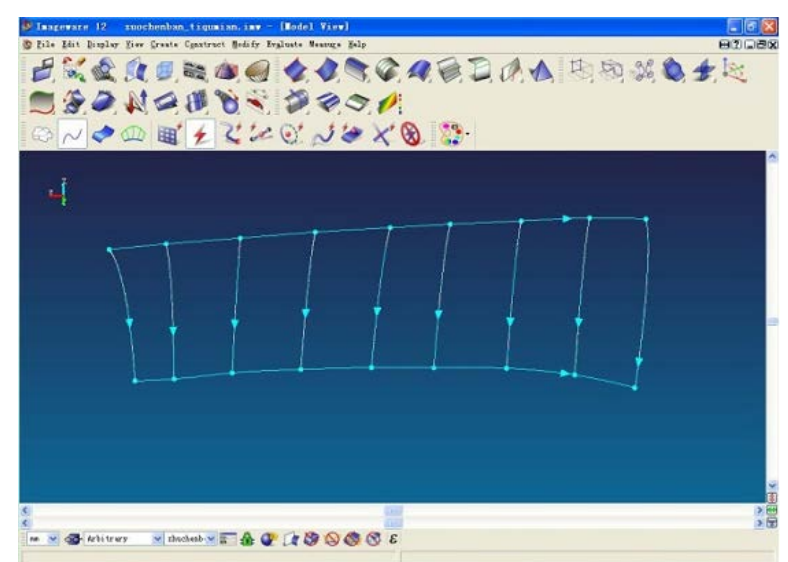

Fig. 6 characteristic line intercept from large surface

Most of the curve Constructed could not be used to construct surface directly, and need revision according to the actual situation, such as clip, segmentation, extension and connection of the curve, etc. Figure 6 -- 8 shows respectively the boundary curve and section characteristic curve of each lining board curve bunch after Fitting and revising. 


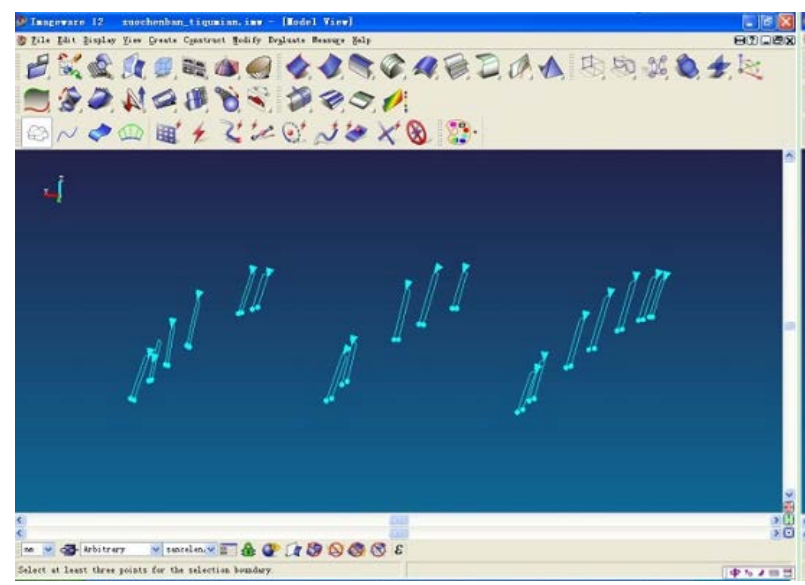

Fig. 7 three chamfered edge section line

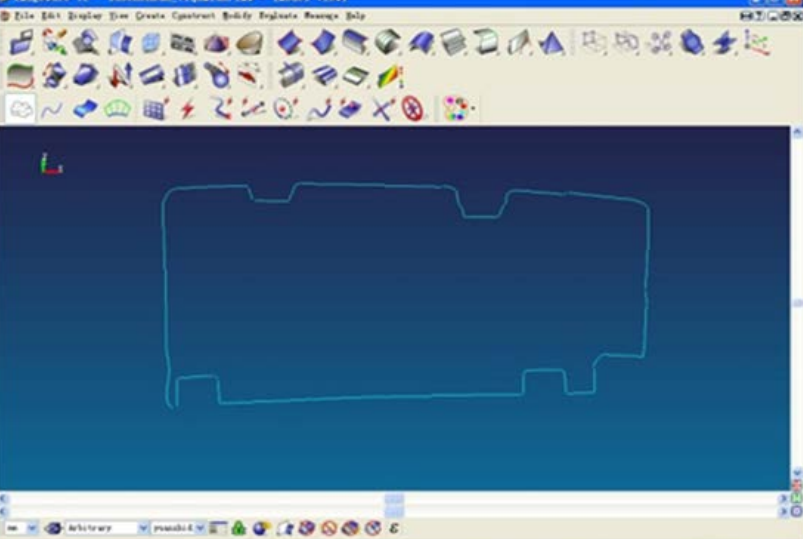

Fig. 8 extracting the boundary curve

Surface construction and revision. Using the Imageware software to do reverse processing, the procedure of data process follows the principle of "point, curve, surface". After adjusting the direction and starting point position of the curve to be consistent, Curve surfaces can be fitted directly by "Constructed/Surface/Loft" function in Imageware. This function is mainly to create a NURBS surface using a set of independent 3-d curve. First select all the curve need fitting, and then choose the continuity of the starting point, choose the direction of the tangent and choose the continuity of end point, finally carries on the curved surface fitting.

After the reconstruction, the single sheet surface also needs some kinds of revision such as surface trimming, splicing, chamfering and so on.

The surface of a complex workpiece not only consists of only one curved surface, but usually of a few or even a dozen curved surface pieces in different sizes. Surface splicing is a rather practical method to reconstruct a complex curved surface. The key of parametric surface splicing is the continuity of the boundary. The boundary of adjacent surfaces should be with a first or second order geometrical continuity on the border.

In addition, during surface revision there often appear problems of processing of spatial trilateral surface blocks at the corner of the joint of surfaces, which is one of rather difficult problems in modelling. It is found through persistent research that a closed trilateral surface blocks could be obtained which fit very well with the surrounding curved surfaces, by means of changing the trilateral surface block problem into a question of two quadrangular surface block structure and then getting it trimmed.

Figure 9 and 10 show respectively the left plate middle bedding face and three chamfered edge after fitting and revision.

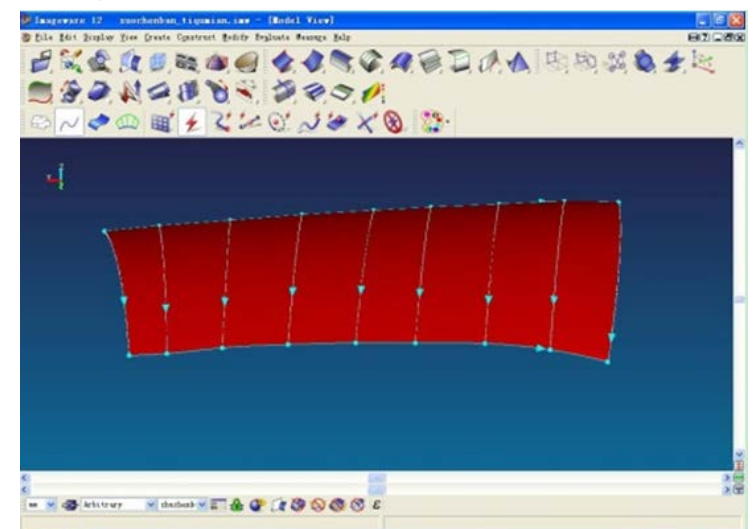

Fig. 9 middle bedding face lofting surface

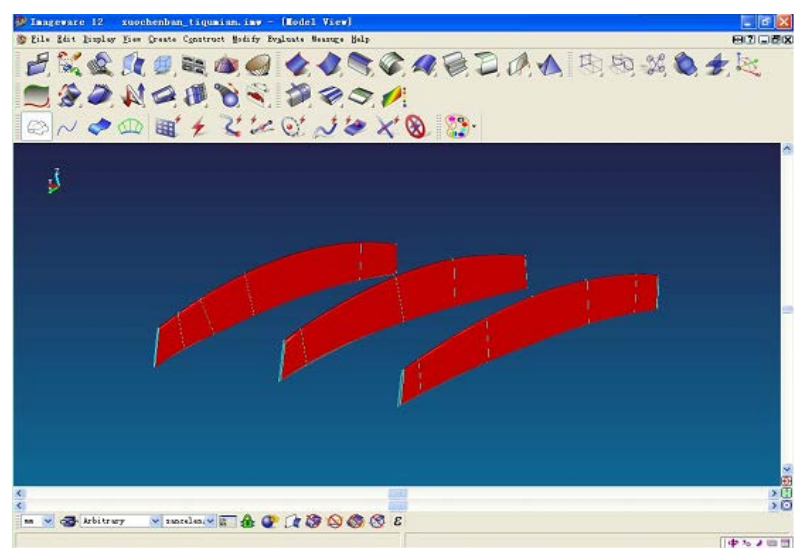

Fig. 10 three chamfered edge lofting surface

Model reconstruction and parameterized in UG. In the UG NX software, “. IMW” format file of Imageware software should be read directly. Import the characteristic curve, plane and curve surface into UG NX, which had been built in Imageware and shall be re-parametrized in UG 
according to the constituted lines and surfaces with ensuring the consistency of coordinate systems. After all these, the import curve should pass through analysis and smoothing treatment, or, reconstitution and editing. On the basis of which and by feature modeling and surface modeling function of UG NX, carry out model reconstruction and redesign and finally 3-d modelling. figure 11shows the 3-d entity modelled by UG NX.

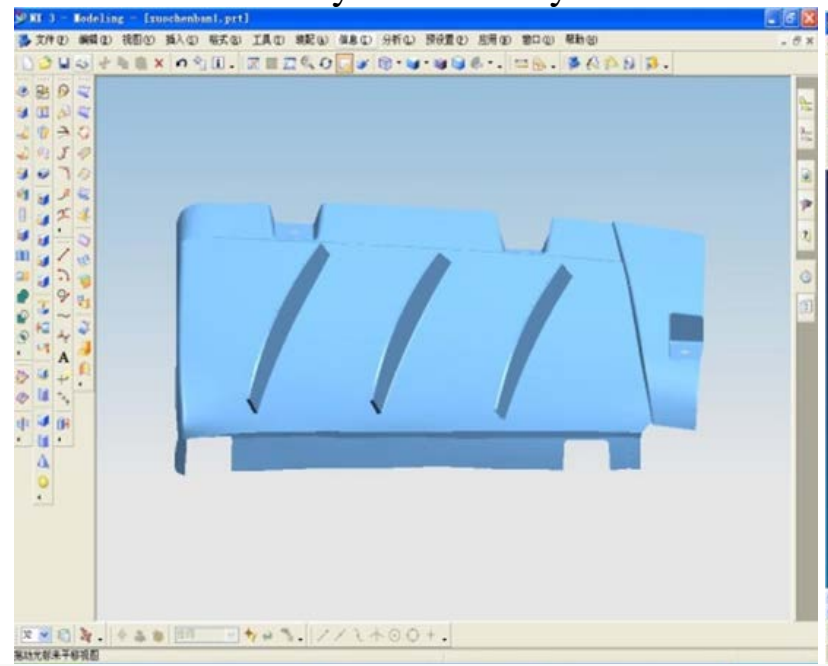

Fig. 11 complete entity modelling

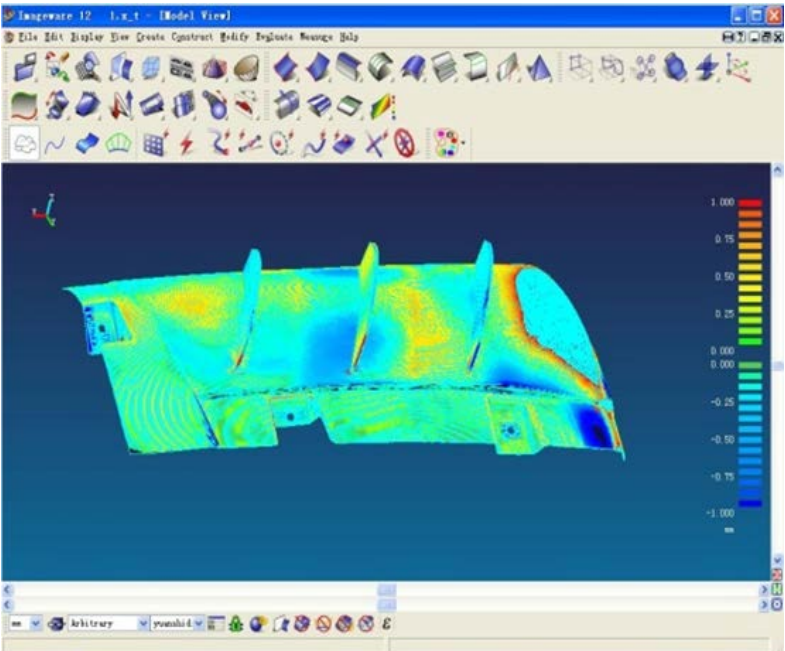

Fig. 12 figure of test and contrast

Error inspection. The 3-d modelling reconstructed with UG NX software could be imported into Imageware software in turn after completion of it, to compare and analyse the deviation between the final reconstruction of 3-d model and the scan point clouds. By displaying the deviation using color cloud picture, the deviation between the curve surface and point cloud can be understood very visually. In addition, you can specify an acceptable tolerance zone and find out the number of points within it, to detect the accuracy of reverse scanning measurement. The result of detection and comparison is shown in figure 12.

\section{Conclusions}

By taking Left panel-lined (left liner) of a Jiefang heavy truck (J6) as an example, the particular methods are introduced in this paper for each parts in large surface reconstitution using ATOS optical scanners, Imageware and UG NX software, including curve construction, curve surface partitioning, surface construction, cohesion of transitional surface, etc. Although surface reconstruction is facing point cloud data, and professional reverse design software provides a variety of tools as well for point cloud to be composited directly into curve surface. But actually, the bridge role of curve can't be ignored in surfaces adjustment. The idea is especially put forward in this paper to reconstitute curve surface with "characteristic line" as the center. "Characteristic line" is the boundary of curve surface to be reconstituted from point cloud region.

"Characteristic line" is just like the skeleton of surface, by which we can divide the point cloud region. It is not difficult to reconstitute a complete surface model,

just making sure the fitting quality of single surface in the region and surface continuity between $\mathrm{m}$ ore surface areas(where the "characteristic line" locates). Further more, the modeling time is greatly reduced.

\section{References}

[1] X.P. Cheng, L. Li, K Zhang.. Reverse engineering technology in the application of water pump impeller measurement and processing. CAD/CAM and manufacturing information, 2004,12.50-52.

[2] R. Mo. Product 3 d CAD tools Unigraphics NX basis and application, Mechanical Industry

Publishing House, 2004 
[3] Y.Q. Jiang, P.J. Liu. Textbook of UG/Imageware reverse engineering training, Tsinghua University Press, 2004

[4] H.Y. Zhu, Z.L. Sun. The application of reverse engineering technology, Henan productivity promotion center, 2002

[5] J. Wu. Huazhong University of Science and Technology, Automobile cover parts mould design system based on living example (master's thesis)

[6] Sun Fuhui - BUAA 20010801 -- Some key technology research of CAD model reconstruction in reverse engineering (master's thesis)

[7] J.K. Ruan, X.L. Zhou, Z.J. Dai, Application of surfacer in automobile body covering parts reverse engineering. Mould Industry. 2004.

[8] Y.Q. Guang. Jilin University. Reverse engineering key technologies and application studies facing to multi-point figuration (master's thesis)

[9] H.M. Ceng, Chongqing University, surface reconstruction technology research in reverse engineering (master's thesis)

[10] S.W. Zheng. Southeast University, Curve and surface reconstruction studies in reverse engineering (master's thesis) 